\title{
A systematic review on the effect of bevacizumab in exudative age-related macular degeneration: a response to a letter to the editor by Schmucker et al.
}

\author{
Jan S. A. G. Schouten • Ellen C. La Heij • \\ Carroll A. B. Webers • Igor J. Lundqvist • \\ Fred Hendrikse
}

Received: 5 November 2009 / Accepted: 6 November 2009/Published online: 8 December 2009

(C) The Author(s) 2009. This article is published with open access at Springerlink.com

The letter of Schmucker et al. is much appreciated. It reflects their careful reading of our article. They address one of the major issues in medicine: what constitutes the evidence base of medicine, and when should doctors start treating patients? We would like to reflect on this issue, and address some of the points raised by Schmucker et al.

The case of bevacizumab has strong similarities with the discovery of insulin. In 1922, Leonard Thompson was the first patient who received insulin [1]. The effects were spectacular. No one had any reasonable doubt that this was due to insulin. Without any randomised controlled trial, insulin has changed the life of many patients with diabetes.

The authors state that "It is common knowledge that due to a missing control group no conclusions on efficacy can be drawn from case series (refers to before-and-after studies in the review by Schouten et al.)." We, and especially one of us who is an associate professor in clinical epidemiology (JS) are well aware of this common knowledge. However, when the issue of whether the observed change in visual acuity in the before-and-after studies is caused by bevacizumab is addressed, prior knowledge should be taken into account. Schmucker et al. will recognise this as a "Bayesian approach".

The authors had no commercial or proprietary interest.

J. S. A. G. Schouten $(\bowtie) \cdot$ E. C. La Heij · C. A. B. Webers • I. J. Lundqvist $\cdot$ F. Hendrikse

Department of Ophthalmology, Maastricht University Hospital, PO box 5800, 6202 AZ Maastricht, The Netherlands

e-mail: Jschou@soog.azm.nl
This prior knowledge constitutes the results from other studies like those on:

- the reported increase of VEGF in AMD,

- the inhibitory effect of VEGF on the formation of new vessels,

- the inhibitory effect of VEGFs on an occurrence of leakage in these vessels,

- basic science on the structure of bevacizumab and its blocking effect of VEGFs,

- the levels of free VEGF in the eye after intravitreal administration of bevacizumab,

- its positive effect in other diseases with the same pathogenesis of neovascularisation such as cancer,

- the results of an RCT on the effect on visual acuity of an almost similar molecule with the same property of blocking VEGFs (ranibizumab).

In addition to the knowledge from other studies, experience from ophthalmologists who treat patients with AMD should be taken into account. One may not take this as solid scientific evidence, but it contributes to the prior view on the effect of bevacizumab. The case of bevacizumab is similar to the case of insulin when one has seen the disappearance of neovascularisation, e.g. in the case of rubeosis of the iris or the disappearance of macular fluid after the injection of bevacizumab.

The issues of methodological shortcomings as addressed by Schmucker et al. are much appreciated, but as good clinicians we need to decide whether the observed change in visual acuity is due to the drug or bother about possible methodological shortcomings. The prior odds are against an explanation by methodological shortcomings. Moreover, we have addressed this issue in a formal way by subgroup analysis based on quality characteristics of the studies. No 
large differences between subgroups were observed. Based on our experience as ophthalmologists, the major methodological issue that may cause a bias is, to our opinion, the way the assessment of the visual acuity was conducted. This was mentioned in our review.

There is always room for doubt, but one has to decide whether there is any reasonable doubt. Ultimately, the question should be answeredas to whether patients with AMD should be treated with bevacizumab or not. Or should we decide that the observed change in visual acuity is due to methodological shortcomings? Given our current knowledge, in its broadest sense, can we withhold this treatment from patients? Should we wait for a randomised placebo controlled trial?

The definition of evidence-based medicine is: "the judicious use of the best current evidence in making decisions about the care of the individual patient. Evidence-based medicine (EBM) is a means to integrate clinical expertise with the best available research evidence and patient values." The most important word is "judicious"; it also reflects the most difficult part.

Safety of a drug is another issue raised by Schmucker et al. First and foremost, the safety of a drug can hardly, if ever be proved. Observed, presumed adverse events can be reported, and the incidence be calculated. For that reason, we did not conclude whether bevacizumab was safe, as is often stated in other studies, but confined ourselves to the incidence of adverse events.

Cases and case series of observed presumed adverse events show the possible adverse events; their risk can not be calculated. The incidence we calculated was based on the reported adverse events in the cited articles that were used in the systematic review. We deliberately spoke about reported adverse events.

We did not limit ourselves to these studies. We compared them with the calculated incidence from the data of the International Intravitreal Bevacizumab Safety Survey [2]. This registration may not have complete data. For that reason, we also compared the results with the data from a prospective follow-up study with careful follow-up of 1,265 consecutive patients with various diagnoses, including exudative AMD, who received 4,303 intravitreal injections of bevacizumab [3]. The follow-up to discover adverse events was thorough. As stated by the authors: "Monitored systemic conditions included myocardial infarction, stroke, systemic hypertension, thromboembolic diseases and death. Blood pressure was measured prior to bevacizumab injection and at 2 weeks following each injection. Other systemic conditions were assessed by a thorough review of systems. All patients were contacted in December 2006 and January 2007, and asked to return for a check-up. If the patients were unable to attend, a telephone interview was conducted to assess for possible systemic complications." The reported incidences were mentioned explicitly in our article.

Compared with these studies, the observed adverse events and the incidences in the studies included in the systematic review were not that different.

Again, the prior odds of developing systemic adverse events could be considerable if taking into account the adverse events that occur when bevacizumab is given intravenously. However, the dosage given in the case of cancer ranges from 5 to $15 \mathrm{mg} / \mathrm{kg}$. The dosage for an intravitreal injection is usually $1.25 \mathrm{mg}$. This is about $1 / 300$ to $1 / 1000$ the dose given in patients with cancer. Moreover, the dose is given in a compartment (the eye) and not intravenously. The much lower dose and injection in a compartment make it a priori less likely that dose-dependent systemic adverse events as seen in cancer patients will occur to the same extent as after intravenous injections.

Since bevacizumab is used frequently over the whole world, and is used in many studies in humans, we have much experience with intravitreous bevacizumab and we know much about its adverse events.

Safety can never be guaranteed, but the adverse events and their incidence should be weighed against its benefit. In the case of AMD, the balance is in favour of giving bevacizumab.

We agree with Schmucker et al. that the longterm safety of bevacizumab is not yet known. But there is only one way to discover this, and that is by giving bevacizumab. Post-marketing surveillance is essential.

Open Access This article is distributed under the terms of the Creative Commons Attribution Noncommercial License which permits any noncommercial use, distribution, and reproduction in any medium, provided the original author(s) and source are credited.

\section{References}

1. http://www.discoveryofinsulin.com/Home.htm

2. Fung AE, Rosenfeld PJ, Reichel E (2006) The international intravitreal bevacizumab safety survey: using the internet to assess drug safety worldwide. Br J Ophthalmol 90:1344-1349

3. Wu L, Martinez-Castellanos MA, Quiroz-Mercado H, Arevalo JF, Berrocal MH, Farah ME, Maia M, Roca JA, Rodriguez FJ, PACORES (2008) Twelve-month safety of intravitreal injections of bevacizumab (Avastin): results of the Pan-American Collaborative Retina Study Group (PACORES). Graefes Arch Clin Exp Ophthalmol 246:81-87 